\title{
Chimera State in the Network of Fractional-Order FitzHugh-Nagumo Neurons
}

\author{
Janarthanan Ramadoss, ${ }^{1}$ Sajedeh Aghababaei ${ }^{D},{ }^{2}$ Fatemeh Parastesh (D), \\ Karthikeyan Rajagopal $\mathbb{D}^{3},{ }^{3}$ Sajad Jafari $\mathbb{D}^{2,4}$ and Iqtadar Hussain $\mathbb{D}^{5}$ \\ ${ }^{1}$ Centre for Artificial Intelligence, Chennai Institute of Technology, Malayambakkam, India \\ ${ }^{2}$ Department of Biomedical Engineering, Amirkabir University of Technology, No. 350, Hafez Ave, Valiasr Square, \\ Tehran 159163-4311, Iran \\ ${ }^{3}$ Centre for Nonlinear Systems, Chennai Institute of Technology, Malayambakkam, India \\ ${ }^{4}$ Health Technology Research Institute, Amirkabir University of Technology, No. 350, Hafez Ave, Valiasr Square, \\ Tehran 159163-4311, Iran \\ ${ }^{5}$ Department of Mathematics, Statistics and Physics, Qatar University, Doha 2713, Qatar
}

Correspondence should be addressed to Karthikeyan Rajagopal; rkarthiekeyan@gmail.com

Received 13 May 2021; Accepted 21 June 2021; Published 30 June 2021

Academic Editor: Guillermo Huerta Cuellar

Copyright ( 2021 Janarthanan Ramadoss et al. This is an open access article distributed under the Creative Commons Attribution License, which permits unrestricted use, distribution, and reproduction in any medium, provided the original work is properly cited.

\begin{abstract}
The fractional calculus in the neuronal models provides the memory properties. In the fractional-order neuronal model, the dynamics of the neuron depends on the derivative order, which can produce various types of memory-dependent dynamics. In this paper, the behaviors of the coupled fractional-order FitzHugh-Nagumo neurons are investigated. The effects of the coupling strength and the derivative order are under consideration. It is revealed that the level of the synchronization is decreased by decreasing the derivative order, and the chimera state emerges for stronger couplings. Furthermore, the patterns of the formed chimeras rely on the order of the derivatives.
\end{abstract}

\section{Introduction}

Fractional-order models have attracted much attention from scientists in different fields such as physics and electronics [1-5]. Considering the fractional derivative, a memory feature is added to the systems. Therefore, the fractional-order model can provide a more precise description of the real phenomena than the integer-order [6]. Furthermore, the fractional calculus has found wide applications in controlling the integer-order systems [7]. The fractional derivative also plays important role in demonstrating different firing patterns of the neurons [8]. Consequently, several fractional neuron models have been presented [9-11].

The complicated interactions among the neurons cause the neural system to act as a complex network [12]. The emergence of collective behaviors is an important characteristic of complex networks [13]. Some examples of collective behaviors are synchronization [14], chimera state [15], and solitary state [16]. Synchronization is an important phenomenon in many applications [17]. Many studies have focused on the synchronization of chaotic systems [18-23]. Furthermore, synchronization manages many neural functions and participates in many brain disorders [24]. In special cases, synchrony and asynchrony are observed simultaneously in a specific region of the brain. For example, the unihemispheric sleep, the neural bump state, and the epileptic seizure disease can be mentioned [25]. This particular condition is called the chimera state [26]. After the foundation of chimera state in 2002 [27], it became the focus of many researchers in a variety of dynamical systems such as the mechanical [28], optical [29], and chemical [30] oscillators and neuronal models [25, 31, 32]. Furthermore, these studies have represented the chimeras with different 
spatiotemporal patterns and properties, including the amplitude chimera [33] and traveling chimera [34].

In neuronal studies, the chimeras were under consideration from different perspectives such as the neurons' dynamics, network topology, and coupling scheme. Santos et al. [35] investigated the chimera in 2D networks with regular and fractal topologies and found the spiral chimeras with multiple asynchronous cores. Wang et al. [36] reported the existence of chimera state in the hyperchaotic neurons with hyperchaotic dynamics. Blondeau Soh et al. [37] represented that shifting the neighbors in the coupling leads the network towards the chimera state. Provata and Venetis [38] studied a neuronal network with power-law coupling and showed that the chimera exists in the weak couplings with large exponents. Li et al. [39] considered two unidirectionally coupled layers of neurons and showed different collective behaviors in the master layer and the induced imperfect chimera state in the slave layer.

Among the chimera studies in neuronal networks, a few have considered the fractional models. Vázquez-Guerrero et al. [40] showed that the network of fractional Hindmarsh-Rose neurons is capable of representing chimera state. They also presented a fractional adaptive controller to obtain the synchronization. In another study, they designed an observer to synchronize the chimera state in coupled fractional neurons [41]. He [42] investigated the magnetic Hindmarsh-Rose model with fractional derivative and showed that analyzing the complexity of the network can help in recognition of its dynamical behavior. In this paper, we study the dynamical behaviors of a network of fractionalorder FitzHugh-Nagumo systems. The effects of the order of derivatives and the coupling strength on the chimera state are under consideration.

\section{The Model}

Recently, the scientists have focused on proposing new models for describing the neural behaviors with considering different aspects of neurons $[43,44]$. Here, we use the FitzHugh-Nagumo (FHN) model with considering the fractional derivative as follows [45]:

$$
\begin{aligned}
& \frac{\mathrm{d}^{q} u}{\mathrm{~d} t^{q}}=u-\frac{u^{3}}{3}-v+I, \\
& \frac{\mathrm{d}^{q} v}{\mathrm{~d} t^{q}}=0.08(u+0.7-0.8 v),
\end{aligned}
$$

where $u$ and $v$ are the membrane voltage and the recovery variable and $I$ is the external excitation current fixed at 0.5 . The fractional derivative order is denoted by $q$, and $\mathrm{d}^{q} / \mathrm{d} t^{q}$ is the Caputo-Fabrizio (CF) fractional operator defined by

$$
\frac{\mathrm{d}^{q}}{\mathrm{~d} t^{q}} u(t)=\frac{1}{\Gamma(1-q)} \int_{t_{0}}^{t} \frac{\dot{x}(\tau)}{(t-\tau)^{q}} \mathrm{~d} \tau, \quad 0<q<1,
$$

where $\Gamma$ is the Gamma function. The dynamics of the model relies on the values of the derivative order. Figure 1(a) represents the bifurcation of the model according to $q$. To consider the spiking firing for the model, the range of $0.7<q<1$ is selected in all simulations. The time series and phase spaces of the model for $q=1,0.9,0.8$, and 0.7 are shown in Figures 1(b)-1(e). It is observed that by decreasing $q$, the amplitude of the oscillations decreases and the period increases.

We consider the network of fractional FHN neurons with the following equations:

$$
\begin{aligned}
& \frac{\mathrm{d}^{q} u_{i}}{\mathrm{~d} t^{q}}=u_{i}-\frac{u_{i}^{3}}{3}-v_{i}+I+d \sum_{j=1}^{N} G_{i j}\left[b_{u u}\left(u_{j}-u_{i}\right)+b_{u v}\left(v_{j}-v_{i}\right)\right], \\
& \frac{\mathrm{d}^{q} v_{i}}{\mathrm{~d} t^{q}}=0.08\left(u_{i}+0.7-0.8 v_{i}\right)+d \sum_{j=1}^{N} G_{i j}\left[b_{v u}\left(u_{j}-u_{i}\right)+b_{v v}\left(v_{j}-v_{i}\right)\right],
\end{aligned}
$$

where $d$ is the coupling strength and $G$ is the Laplacian matrix of connections. The network has a ring structure with nonlocal coupling as shown in Figure 2 (each neuron is connected to its 40 nearest neighbors, and $N=100$ ). The coupling between variables is through a rotational matrix as follows [46]:

$$
B=\left(\begin{array}{ll}
b_{u u} & b_{u v} \\
b_{v u} & b_{v v}
\end{array}\right)=\left(\begin{array}{cc}
\cos \phi & \sin \phi \\
-\sin \phi & \cos \phi
\end{array}\right),
$$

with $\phi=(\pi / 2)-0.1$ being the coupling phase.

\section{Results}

The network is solved numerically by using the Adam-Bashforth method based on the algorithm proposed in [47] with random initial conditions. To identify different behaviors, the strength of incoherence (SI) is used [48]. To find this measure, at first, the variables are transformed into new ones as $x_{i}=u_{i}-u_{i+1}, i=1, \ldots, N$. Then, the network is divided into $M=N / n$ groups of $n$ neurons, and the local standard deviation is computed as follows:

$$
\sigma(m)=\left\langle\sqrt{\frac{1}{n} \sum_{j=n(m-1)+1}^{m n}\left[x_{i}-\langle x\rangle\right]^{2}}\right\rangle, \quad m=1, \ldots, M,
$$

where $\langle x\rangle=1 / N \sum_{i=1}^{N} x_{i}(t)$. Finally, the SI is computed by

$$
\begin{gathered}
s_{m}=\theta(\delta-\sigma(m)), \\
\mathrm{SI}=1-\frac{\sum_{m=1}^{M} s_{m}}{M},
\end{gathered}
$$

where $\theta$ is the Heaviside function and $\delta$ is a threshold set at 0.23 , here, and $n=4$. The value of SI determines the behavior of the network by SI $=0,0<\mathrm{SI}<1$, and SI $=1$ for synchronization, chimera, and asynchronization, respectively.

The network of equation (3) with integer-order $(q=1)$ represents different dynamical behaviors with varying the coupling strength $(d)$. However, the dynamical changes occur in very small coupling strengths. The patterns of the neurons by varying $d$ values are demonstrated in Figure 3 . The left panel represents the space-time plots, and the right 


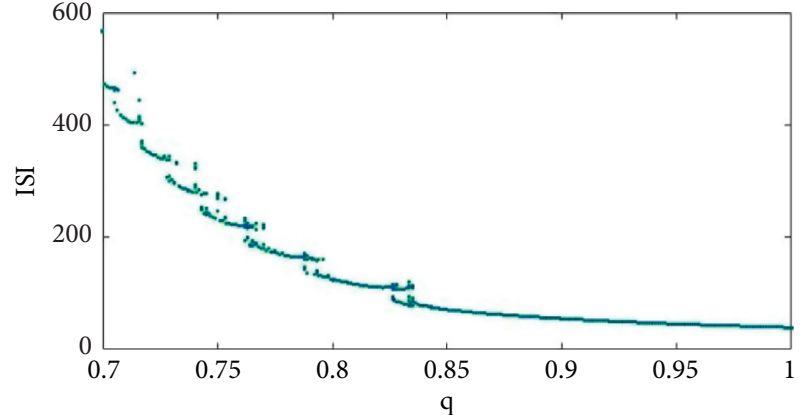

(a)
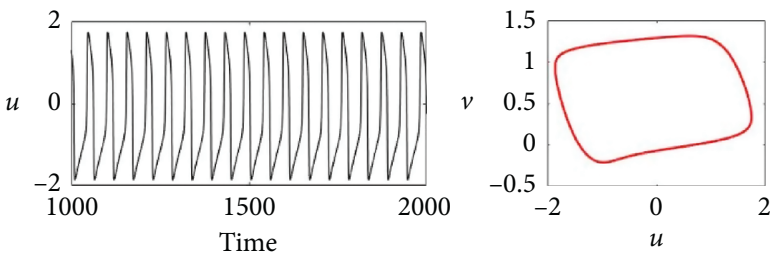

(c)
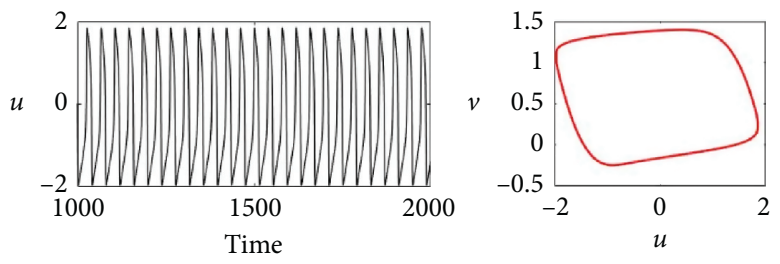

(b)
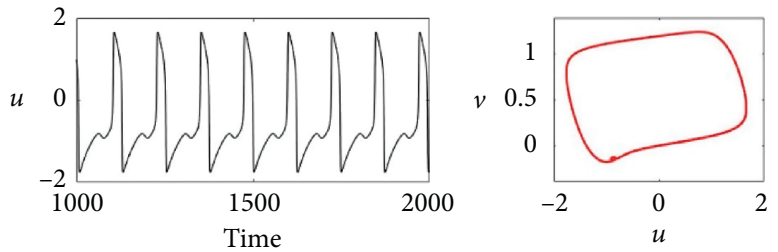

(d)
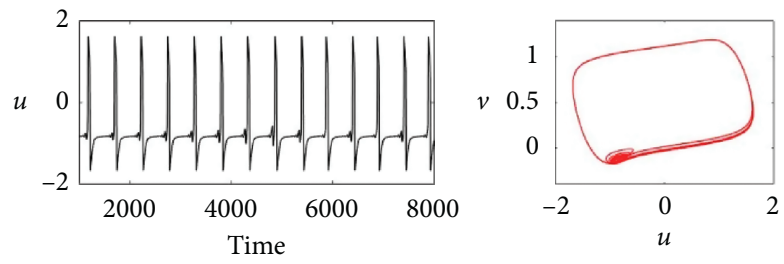

(e)

FIgURE 1: (a) The bifurcation diagram of the model; inter spike intervals (ISI) versus the derivative order $(q)$. (b-e) The time series and phase spaces of the model for different $q$ values: (b) $q=1$; (c) $q=0.9$; (d) $q=0.8$; (e) $q=0.7$.



FIGURE 2: The ring structure of the $N=100$ neurons with nonlocal coupling. Each neuron is connected to its 40 nearest neighbors. The connections for $i=1$ are illustrated.

panel represents the time snapshots of the neurons. By increasing the coupling strength, the initial asynchronous pattern of the neurons changes to the chimera state. For $d=10^{-7}$, a chimera state is created (shown in Figure 3(a)), and it can be seen that there are synchronous and asynchronous neurons in the network. With an increment in the coupling strength, more neurons locate in the synchronous group. The behavior of the network for $d=1.7 \times 10^{-5}$ is illustrated in Figure 3(b). In this case, most neurons are synchronous, while a few oscillate differently. This behavior is called the solitary state $[49,50]$. As the coupling becomes stronger, different neurons are attracted to the synchronous group and a complete synchronization is observed (Figure 3(c)).

The behavior of the neurons is considerably influenced when the derivative of the network's equations changes to the fractional. To investigate this, the coupling strength is considered to be fixed at $d=2 \times 10^{-5}$, where the integerorder network shows the solitary state (similar to Figure 3(b)) and the fractional order is changed. With decreasing the fractional order $(q)$, firstly, the network synchronization is enhanced. This is shown in Figure 4(a) for $q=0.9$. However, more decrement of $q$ disturbs the synchronous behavior of the network. Figure 4(b) represents the chimera state for $q=0.8$ with a similar coupling strength value. When $q$ decreases to $q=0.7$, the network becomes completely asynchronous (Figure 4(c)). The waveforms of the neurons in each case are depicted in the right panel of Figure 4.

For different fractional orders, the range of the coupling strength for the appearance of different dynamical behaviors is different. As the fractional order decreases, the chimera 

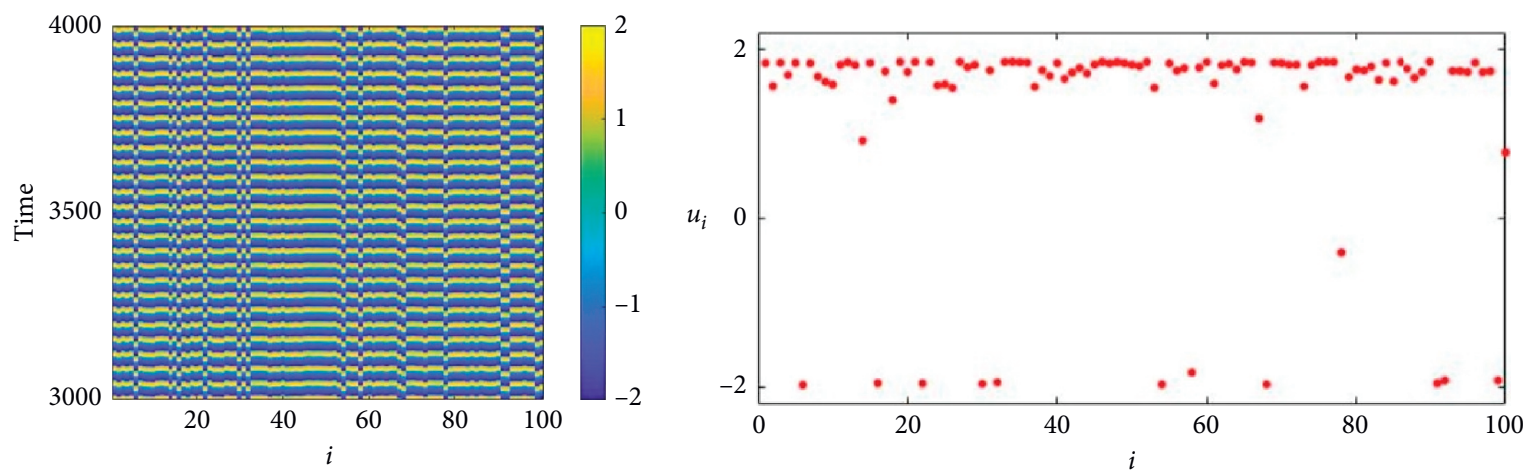

(a)
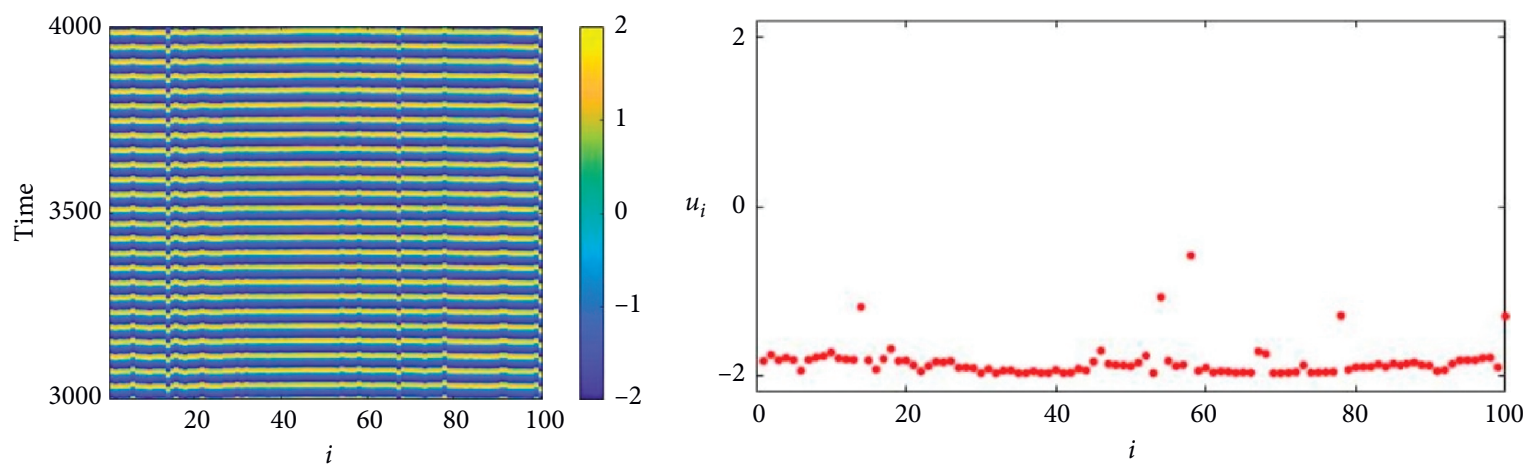

(b)
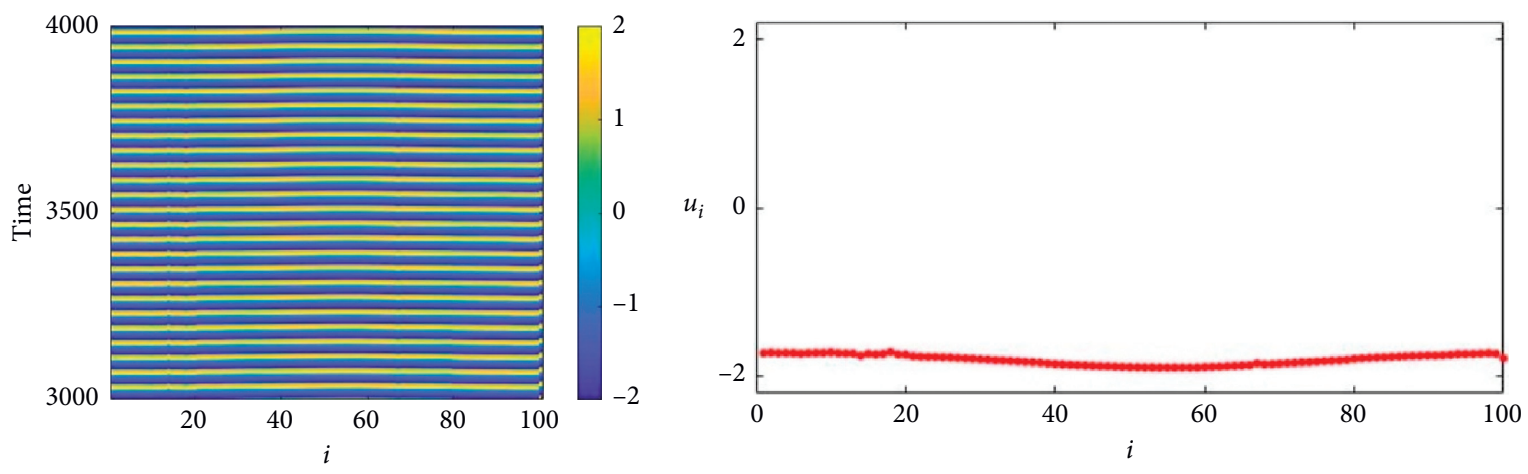

(c)

Figure 3: The patterns of the network (left panel) and time snapshots (right panel) with integer-order derivative: (a) chimera state for $d=10^{-7}(\mathrm{SI}=0.74)$; (b) solitary state for $d=1.7 \times 10^{-5}(\mathrm{SI}=0.39)$; (c) synchronization for $d=5 \times 10^{-5}(\mathrm{SI}=0)$.

state is formed in higher coupling strengths. Figure 5(a) shows the chimera state in $q=0.9$ for $d=4.6 \times 10^{-6}$. In this case, there are several groups containing a few synchronous neurons. For $q=0.8$, the chimera is observed for $d=3 \times 10^{-5}$ and more neurons are involved in the synchronous cluster (Figure 5(b)). When $q$ decreases to $q=0.7$, in some time intervals, some neurons become synchronous. Therefore, the chimera for this derivative is nonstationary. The chimera for $q=0.7$ is shown in Figure 5(c). To illustrate the coherent and incoherent clusters better, the local order parameter is computed and shown in the right column of Figure 5. This parameter can be obtained as $L_{k}=\mid 1 / 2 p \sum_{|k-l| \leq p} \exp$ $\left(j \phi_{l}\right) \mid, k=1, \ldots, N$, where $\phi_{l}$ is the geometric phase of $l$ th oscillator calculated by $\phi_{l}=a \tan \left(y_{l} / x_{l}\right)$. The size of the spatial window is denoted by $p$. When $L_{k}=1$, the $k$ th oscillator belongs to a coherent group.

Figure 6 represents the strength of incoherence of the network for different fractional orders. For $q=1$, the network is in chimera state until $d=5 \times 10^{-5}$ and becomes synchronous for larger coupling strengths (Figure 6(a)). A similar pattern is observed for $q=0.95$ (Figure 6(b)). When $q$ decreases to $q=0.9$, the synchronization occurs for very smaller coupling strengths $\left(d=2.2 \times 10^{-5}\right)$. For $q=0.85$, the network's dynamical behavior returns to the $q=1$ manner. With more decrement of $q$, a stronger coupling is needed for the synchronization. Figure $6(\mathrm{e})$ shows that $q=0.8$ has the larger chimera region. For $q=$ 0.7 and $q=0.75$, a large asynchronization region is 

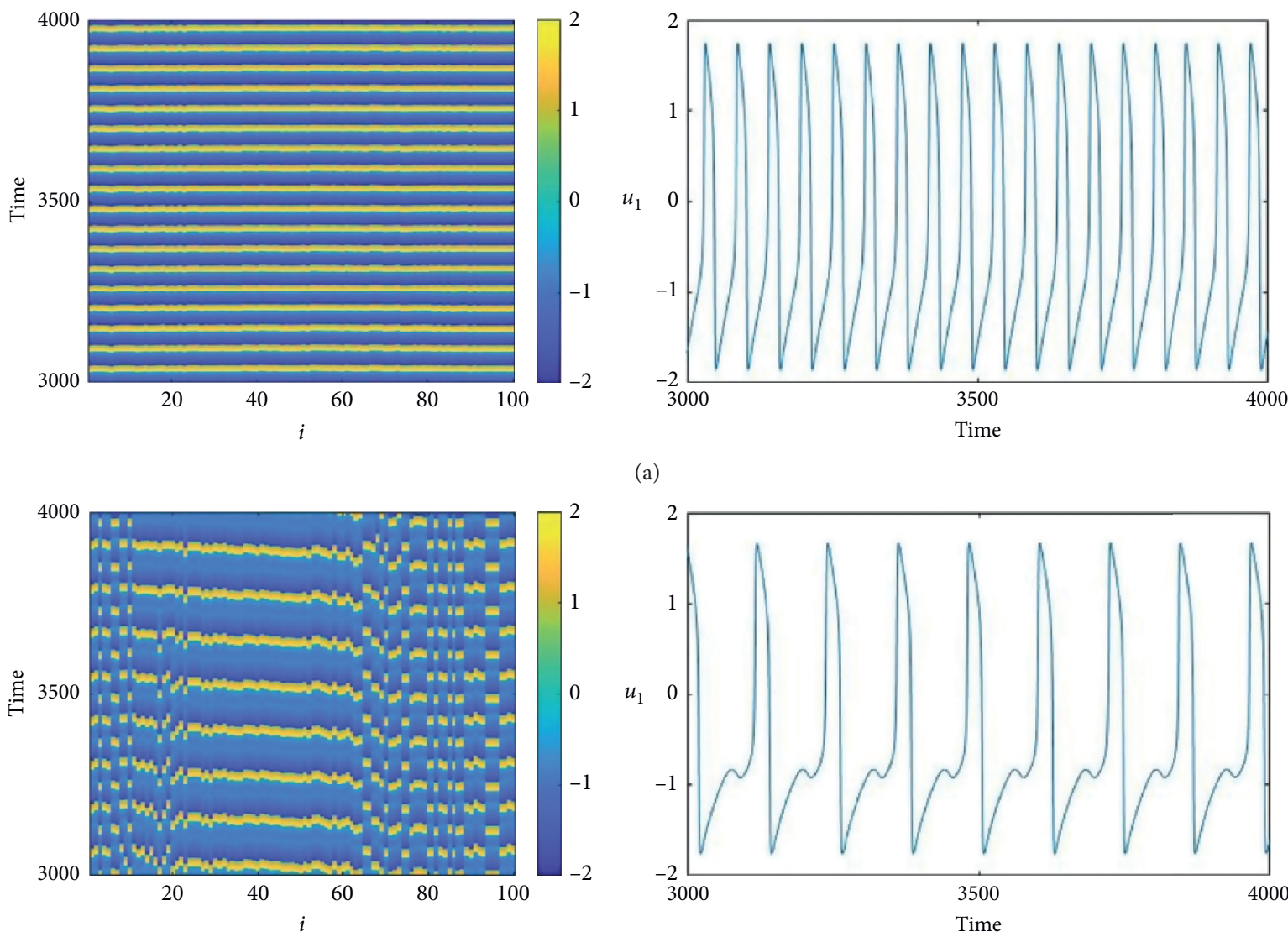

(a)

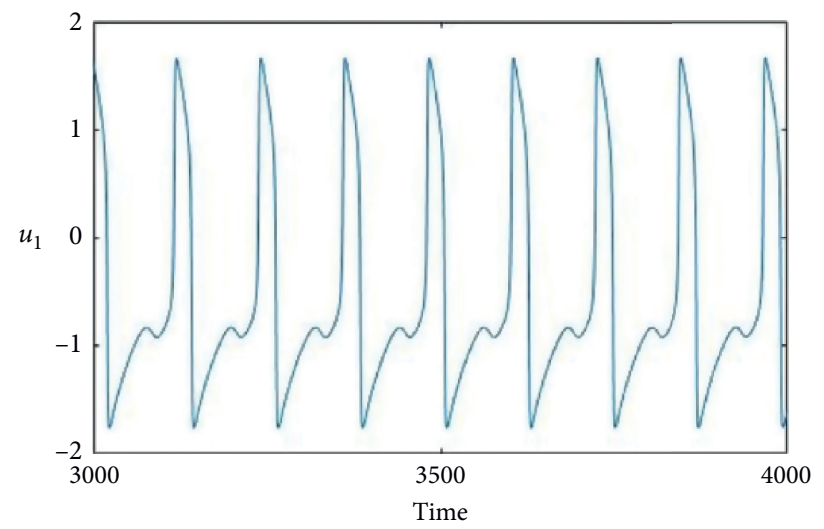

(b)
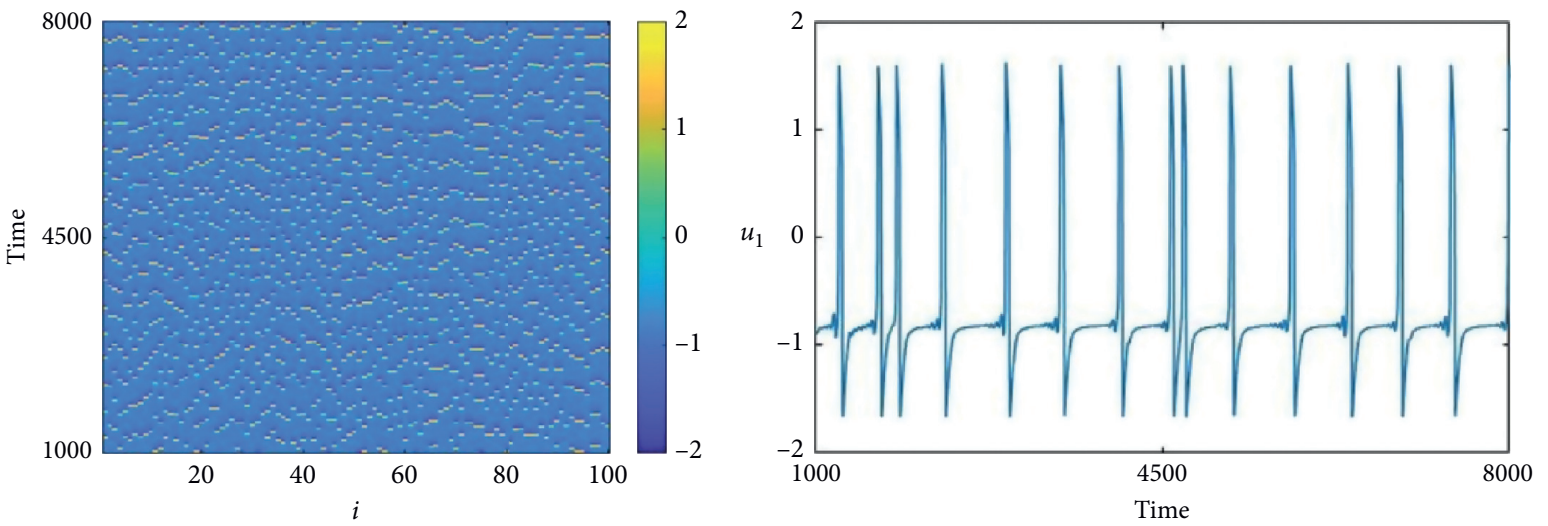

(c)

FIGURE 4: Upper panel: the patterns of the network for $d=2 \times 10^{-5}$ and different derivative orders. The integer-order network exhibits a solitary state at this coupling. (a) Synchronization for $q=0.9(\mathrm{SI}=0)$. (b) Chimera for $q=0.8(\mathrm{SI}=0.8)$. (c) Asynchronization for $q=0.7$ $(\mathrm{SI}=0)$.

observed. The variation of SI according to $q$ is illustrated in Figure 7. It is observed that for low coupling strength, the integer network is in a chimera state. The chimera state is preserved until $q=0.835$, and then all neurons become asynchronous. For strong couplings, the integer-order network is synchronous. With decreasing $q$, the synchronization remains until $q=0.84$. For $q<0.84$, the chimera state is formed. However, in the range $0.8<q<0.823$, the synchronization may appear in the network determined by the initial conditions. 

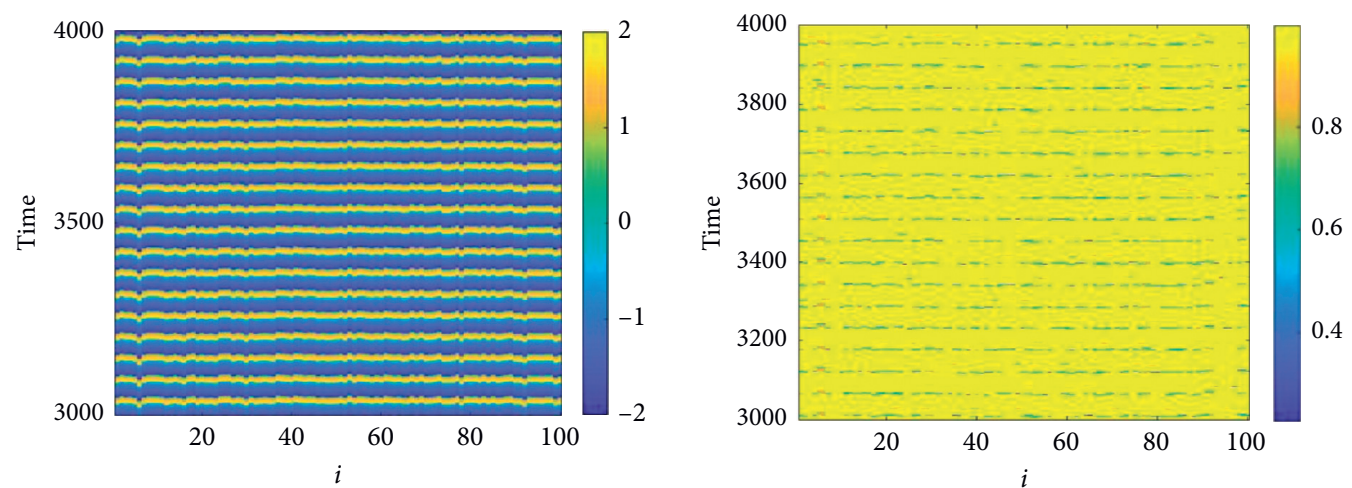

(a)
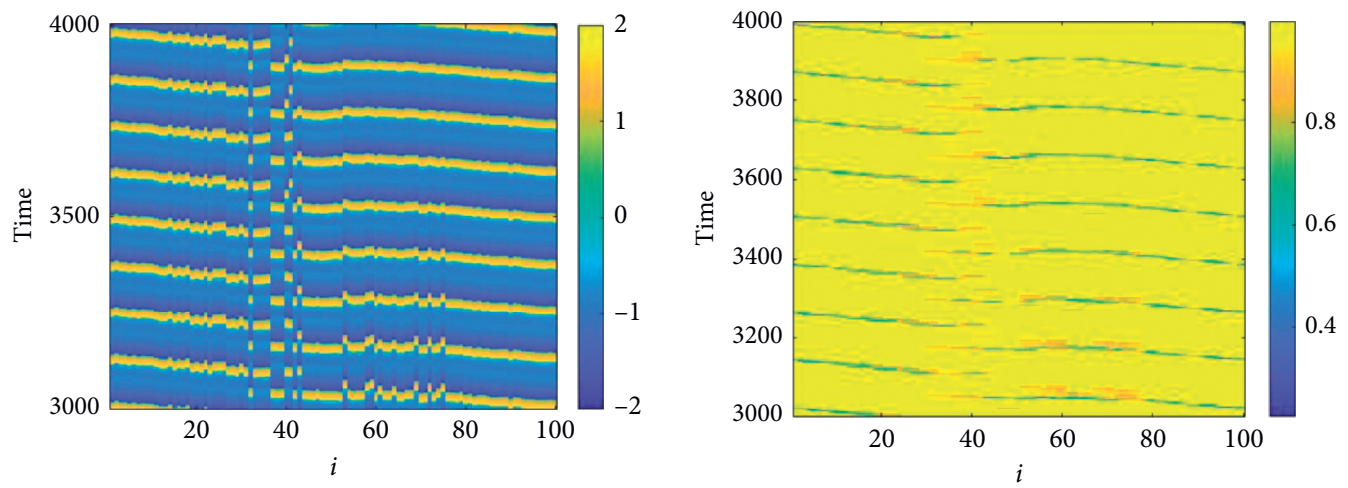

(b)
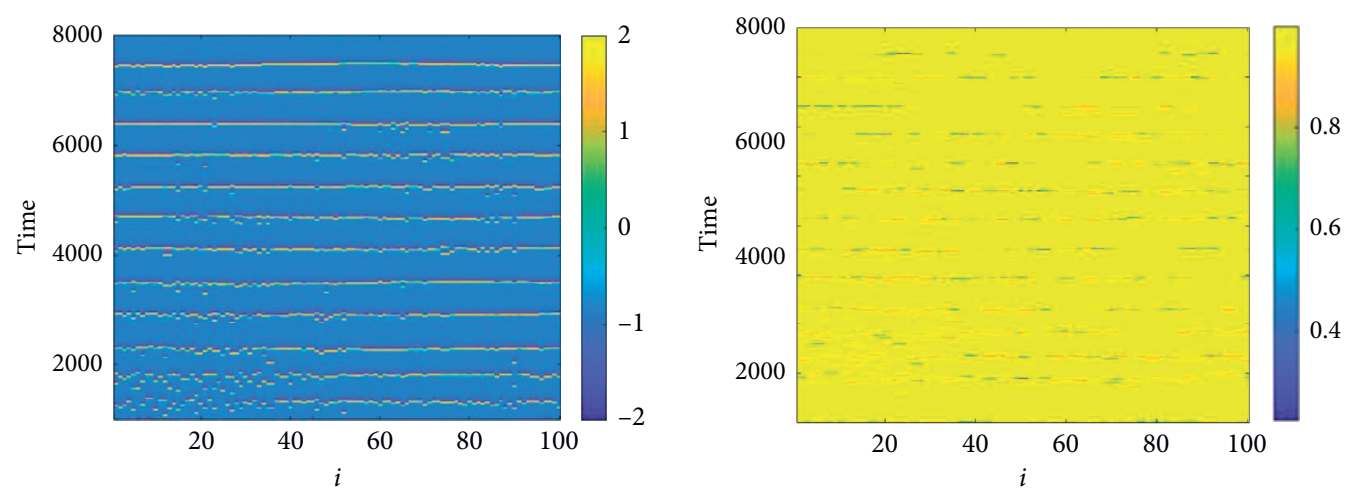

(c)

FIGURE 5: The chimera patterns in the fractional-order network. The left and right columns show the spatiotemporal patterns and the local order parameters $(p=2)$, respectively: (a) $q=0.9$ and $d=4.6 \times 10^{-6}(\mathrm{SI}=)$; (b) $q=0.8$ and $d=3 \times 10^{-5}$ (SI = 0.48); (c) $q=0.7$ and $d=10^{-4}(\mathrm{SI}=0.42)$. By varying the derivative order, the chimera is formed with different patterns.

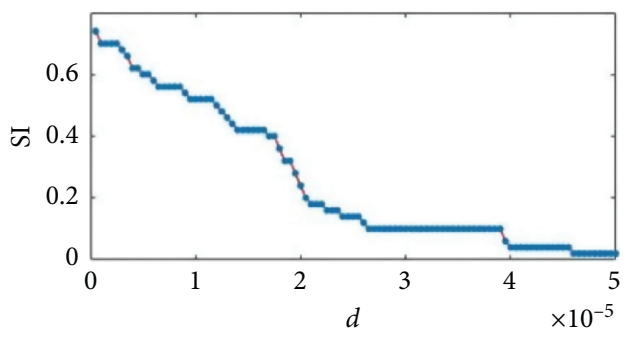

(a)

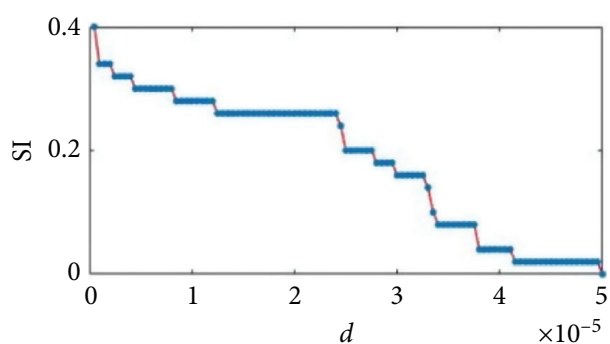

(b)

Figure 6: Continued. 


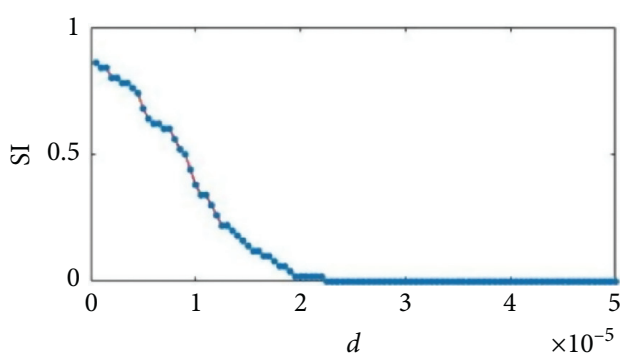

(c)

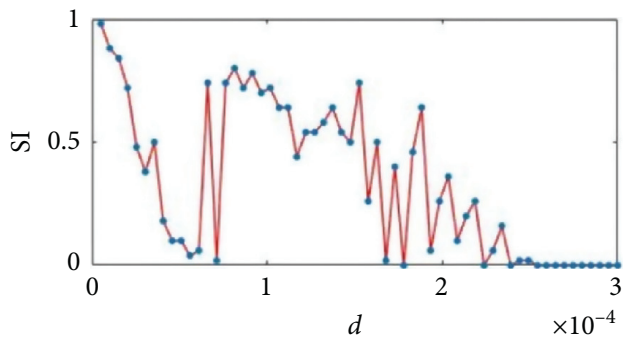

(e)

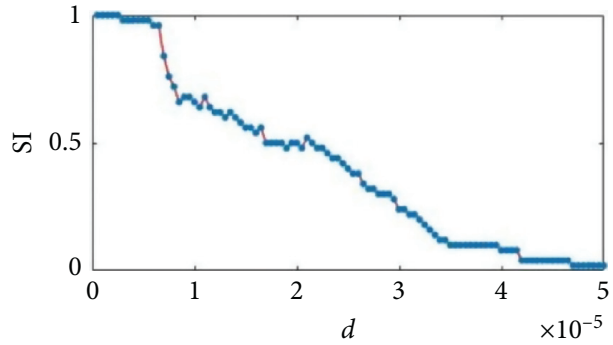

(d)

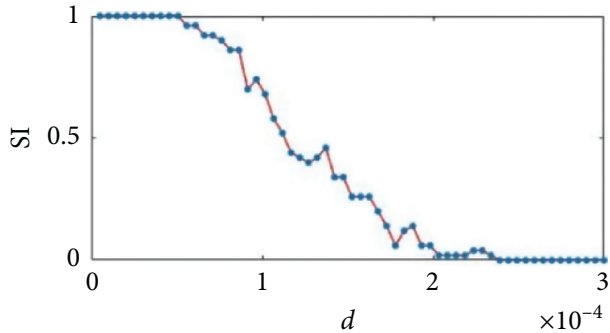

(f)

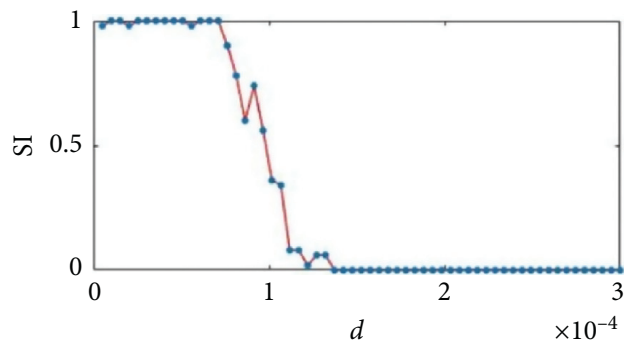

(g)

FIGURE 6: The strength of incoherence according to coupling strength for various values of derivative order: (a) $q=1$; (b) $q=0.95$; (c) $q=0.9$; (d) $q=0.85$; (e) $q=0.8$; (f) $q=0.75$; (g) $q=0.7$.

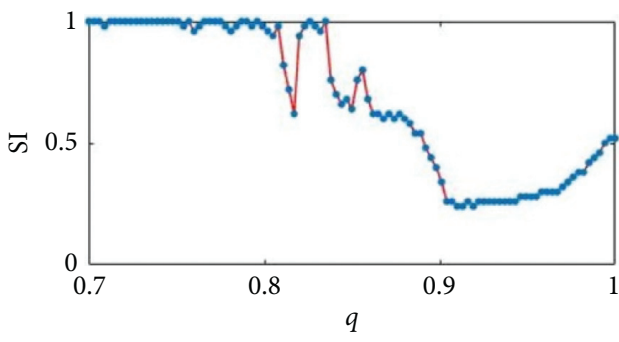

(a)

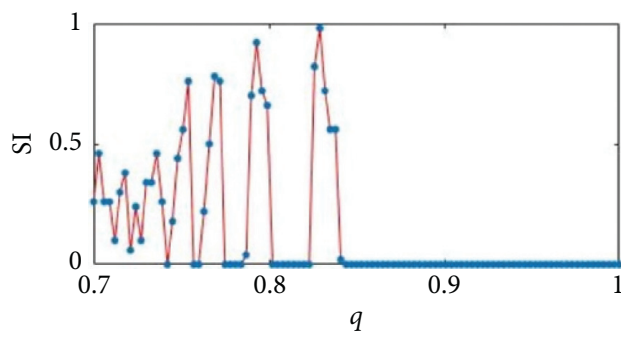

(b)

Figure 7: The strength of incoherence according to derivative order $(q)$ for weak and strong coupling strengths: (a) $d=10^{-5}$; (b) $d=10^{-4}$.

\section{Conclusion}

In this paper, a network of coupled fractional-order FitzHugh-Nagumo neurons was studied. The dynamical behavior of the network was investigated under the variation of the coupling strength and the derivative order. The bifurcation diagram of the fractional system with respect to the derivative order revealed that the dynamics of the model is dependent on the fractional order. Consequently, by changing the value of the derivative order, various collective behaviors of the neurons can be found. The integer-order neurons experience asynchronization, chimera, and synchronization with increasing the coupling strength, respectively. In the fractional-order network, decreasing the derivative order for the constant coupling strength, resulted in the lower synchrony level in the network. Therefore, the fractional-order network has the same state transition; however, it occurs in higher coupling strengths. Thus, the chimera or synchronous states appear for stronger couplings. Furthermore, the pattern of the chimera was changed with varying the derivative order. For $q=0.9$, some small synchronous clusters were formed, while in $q=0.8$, a large 
cluster of synchronous neurons was observed. For lower $\mathrm{q}$ values $(q=0.7)$, the position of the synchronous cluster was time-dependent and the nonstationary chimera state appeared.

\section{Data Availability}

The data used to support the findings of the study are included within the article.

\section{Conflicts of Interest}

The authors declare that there are no conflicts of interest.

\section{Acknowledgments}

This work was funded by the Center for Nonlinear Systems, Chennai Institute of Technology, India, with funding number CIT/CNS/2021/RP-015.

\section{References}

[1] J. L. Echenausía-Monroy, G. Huerta-Cuellar, R. JaimesReátegui et al., "Multistability emergence through fractionalorder-derivatives in a PWL multi-scroll system," Electronics, vol. 9, no. 6, p. 880, 2020.

[2] Y. Peng, S. He, and K. Sun, "Chaos in the discrete memristorbased system with fractional-order difference," Results in Physics, vol. 24, Article ID 104106, 2021.

[3] E. Zambrano-Serrano, E. Campos-Cantón, and J. M. MuñozPacheco, "Strange attractors generated by a fractional order switching system and its topological horseshoe," Nonlinear Dynamics, vol. 83, no. 3, pp. 1629-1641, 2016.

[4] E. Zambrano-Serrano, J. M. Muñoz-Pacheco, and E. CamposCantón, "Chaos generation in fractional-order switched systems and its digital implementation," AEU-International Journal of Electronics and Communications, vol. 79, pp. 43-52, 2017.

[5] S. He, H. Natiq, S. Banerjee, and K. Sun, "Complexity and chimera states in a network of fractional-order laser systems," Symmetry, vol. 13, no. 2, p. 341, 2021.

[6] J.-M. He and F.-Q. Chen, "A new fractional order hyperchaotic Rabinovich system and its dynamical behaviors," International Journal of Non-linear Mechanics, vol. 95, pp. 73-81, 2017.

[7] H. Chen and Y. Chen, "Fractional-order generalized principle of self-support (FOGPSS) in control system design," IEEE/ CAA Journal of Automatica Sinica, vol. 3, no. 4, pp. 430-441, 2016.

[8] S. A. Malik and A. H. Mir, "FPGA realization of fractional order neuron," Applied Mathematical Modelling, vol. 81, pp. $372-385,2020$.

[9] M. Shi and Z. Wang, "Abundant bursting patterns of a fractional-order Morris-Lecar neuron model," Communications in Nonlinear Science and Numerical Simulation, vol. 19, no. 6, pp. 1956-1969, 2014.

[10] A. Mondal, S. K. Sharma, R. K. Upadhyay, and A. Mondal, "Firing activities of a fractional-order FitzHugh-Rinzel bursting neuron model and its coupled dynamics," Scientific Report, vol. 9, pp. 1-11, 2019.

[11] Y. Yu, M. Shi, H. Kang, M. Chen, and B. Bao, "Hidden dynamics in a fractional-order memristive Hindmarsh-Rose model," Nonlinear Dynamics, vol. 100, no. 1, pp. 891-906, 2020.

[12] O. Sporns, "The human connectome: a complex network," Annals of the New York Academy of Sciences, vol. 1224, no. 1, pp. 109-125, 2011.

[13] S. Boccaletti, V. Latora, Y. Moreno, M. Chavez, and D. Hwang, "Complex networks: structure and dynamics," Physics Reports, vol. 424, no. 4-5, pp. 175-308, 2006.

[14] A. Arenas, A. Díaz-Guilera, J. Kurths, Y. Moreno, and C. Zhou, "Synchronization in complex networks," Physics Reports, vol. 469, no. 3, pp. 93-153, 2008.

[15] F. Parastesh, S. Jafari, H. Azarnoush et al., "Chimeras," Physics Report, vol. 898, pp. 1-114, 2020.

[16] E. Rybalova, V. S. Anishchenko, G. I. Strelkova, and A. Zakharova, "Solitary states and solitary state chimera in neural networks," Chaos: An Interdisciplinary Journal of Nonlinear Science, vol. 29, no. 7, Article ID 71106, 2019.

[17] A. Anzo-Hernández, E. Campos-Cantón, and M. Nicol, "Itinerary synchronization between PWL systems coupled with unidirectional links," Communications in Nonlinear Science and Numerical Simulation, vol. 70, pp. 102-124, 2019.

[18] M. Zanin, J. R. Sevilla-Escoboza, R. Jaimes-Reátegui, J. H. Garcia-Lopez, G. Huerta-Cuellar, and A. N. Pisarchik, "Synchronization attack to chaotic communication systems," The Interdisciplinary Journal of Discontinuity, Nonlinearity, and Complexity, vol. 2, no. 4, pp. 333-343, 2013.

[19] D. Lopez Mancilla, E. Villafana Rauda, J. H. Garcia Lopez, R. Jaimes Reategui, G. Huerta Cuellar, and R. Chiu, “Analysis of perturbed synchronization of piecewise rössler using sliding modes control," IEEE Latin America Transactions, vol. 13, no. 8, pp. 2482-2487, 2015.

[20] R. Jaimes-Reátegui, V. P. Vera-Ávila, R. Sevilla-Escoboza et al., "Synchronization of unidirectionally delay-coupled chaotic oscillators with memory," The European Physical Journal Special Topics, vol. 225, no. 13-14, pp. 2707-2715, 2016.

[21] G. Minero-Ramales, D. López-Mancilla, C. E. Castañeda et al., "A discrete-time chaos synchronization system for electronic locking devices," The European Physical Journal Special Topics, vol. 225, no. 13-14, pp. 2655-2667, 2016.

[22] E. Campos, J. Urias, and N. F. Rulkov, "Multimodal synchronization of chaos," Chaos: An Interdisciplinary Journal of Nonlinear Science, vol. 14, no. 1, pp. 48-54, 2004.

[23] J. S. González Salas, E. Campos Cantón, F. C. Ordaz Salazar, and I. Campos Cantón, "Forced synchronization of a selfsustained chaotic oscillator," Chaos: An Interdisciplinary Journal of Nonlinear Science, vol. 18, no. 2, Article ID 23136, 2008.

[24] S. Petkoski and V. K. Jirsa, "Transmission time delays organize the brain network synchronization," Philosophical Transactions of the Royal Society A: Mathematical, Physical and Engineering Sciences, vol. 377, no. 2153, Article ID 20180132, 2019.

[25] S. Majhi, B. K. Bera, D. Ghosh, and M. Perc, "Chimera states in neuronal networks: a review," Physics of Life Reviews, vol. 28, pp. 100-121, 2019.

[26] D. M. Abrams and S. H. Strogatz, "Chimera states for coupled oscillators," Physical Review Letters, vol. 93, no. 17, Article ID 174102, 2004.

[27] Y. Kuramoto and D. Battogtokh, "Coexistence of coherence and incoherence in nonlocally coupled phase oscillators," Nonlinear Phenomena in Complex Systems, vol. 5, pp. 380$385,2002$. 
[28] P. R. Carvalho and M. A. Savi, "Synchronization and chimera state in a mechanical system," Nonlinear Dynamics, vol. 102, pp. 907-925, 2020.

[29] E. A. Viktorov, T. Habruseva, S. P. Hegarty, G. Huyet, and B. Kelleher, "Coherence and incoherence in an optical comb," Physical Review Letters, vol. 112, no. 22, Article ID 224101, 2014.

[30] N. M. Awal, D. Bullara, and I. R. Epstein, "The smallest chimera: periodicity and chaos in a pair of coupled chemical oscillators," Chaos: An Interdisciplinary Journal of Nonlinear Science, vol. 29, no. 1, Article ID 13131, 2019.

[31] S. Majhi, M. Perc, and D. Ghosh, "Chimera states in a multilayer network of coupled and uncoupled neurons," Chaos: An Interdisciplinary Journal of Nonlinear Science, vol. 27, no. 7, Article ID 73109, 2017.

[32] Z. Wang, S. Baruni, F. Parastesh et al., "Chimeras in an adaptive neuronal network with burst-timing-dependent plasticity," Neurocomputing, vol. 406, pp. 117-126, 2020.

[33] T. Banerjee, D. Biswas, D. Ghosh, E. Schöll, and A. Zakharova, "Networks of coupled oscillators: from phase to amplitude chimeras," Chaos: An Interdisciplinary Journal of Nonlinear Science, vol. 28, no. 11, pp. 113-124, 2018.

[34] A. J. Alvarez-Socorro, M. G. Clerc, and N. Verschueren, "Traveling chimera states in continuous media," Communications in Nonlinear Science and Numerical Simulation, vol. 94, Article ID 105559, 2021.

[35] M. S. Santos, P. R. Protachevicz, I. L. Caldas et al., "Spiral wave chimera states in regular and fractal neuronal networks," Journal of Physics: Complexity, vol. 2, no. 1, Article ID 15006, 2020.

[36] S. Wang, S. He, K. Rajagopal, A. Karthikeyan, and K. Sun, "Route to hyperchaos and chimera states in a network of modified Hindmarsh-Rose neuron model with electromagnetic flux and external excitation," The European Physical Journal Special Topics, vol. 229, no. 6-7, pp. 929-942, 2020.

[37] G. Blondeau Soh, P. Louodop, R. Kengne, and R. Tchitnga, "Chimera dynamics in an array of coupled FitzHugh-Nagumo system with shift of close neighbors," Heliyon, vol. 6, no. 4, Article ID e03739, 2020.

[38] A. Provata and I. E. Venetis, "Chimera states in Leaky integrate-and-fire dynamics with power law coupling," European Physical Journal B, vol. 93, pp. 1-9, 2020.

[39] X. Li, T. Xu, and J. Li, "Synchronization and chimera states in a multilayer neuronal network with unidirectional interlayer links," The European Physical Journal Special Topics, vol. 228, no. 11, pp. 2419-2427, 2019.

[40] P. Vázquez-Guerrero, J. F. Gómez-Aguilar, F. Santamaria, and R. F. Escobar-Jiménez, "Synchronization patterns with strong memory adaptive control in networks of coupled neurons with chimera states dynamics," Chaos, Solitons \& Fractals, vol. 128, pp. 167-175, 2019.

[41] P. Vázquez-Guerrero, J. F. Gómez-Aguilar, F. Santamaria, and R. F. Escobar-Jiménez, "Design of a high-gain observer for the synchronization of chimera states in neurons coupled with fractional dynamics," Physica A: Statistical Mechanics and Its Applications, vol. 539, Article ID 122896, 2020.

[42] S. He, "Complexity and chimera states in a ring-coupled fractional-order memristor neural network," Frontiers in Applied Mathematics and Statistics, vol. 6, p. 24, 2020.

[43] N. Wang, G. Zhang, and H. Bao, "Bursting oscillations and coexisting attractors in a simple memristor-capacitor-based chaotic circuit," Nonlinear Dynamics, vol. 97, no. 2, pp. 1477-1494, 2019.
[44] Y. Liu, W.-j. Xu, J. Ma, F. Alzahrani, and A. Hobiny, "A new photosensitive neuron model and its dynamics," Frontiers of Information Technology \& Electronic Engineering, vol. 21, no. 9, pp. 1387-1396, 2020.

[45] M. Armanyos and A. G. Radwan, "Fractional-order fitzhughnagumo and izhikevich neuron models," in Proceedings of the 2016 13th International Conference on Electrical Engineering/ Electronics, Computer, Telecommunications and Information Technology (ECTI-CON), pp. 1-5, Chiang Mai, Thailand, June 2016.

[46] I. Omelchenko, O. E. Omel'chenko, P. Hövel, and E. Schöll, "When nonlocal coupling between oscillators becomes stronger: patched synchrony or multichimera states," Physical Review Letters, vol. 11022, 224101.

[47] K. Diethelm and A. D. Freed, "The FracPECE subroutine for the numerical solution of differential equations of fractional order," Forschung und wissenschaftliches Rechnen, vol. 1999, pp. 57-71, 1998.

[48] R. Gopal, V. Chandrasekar, A. Venkatesan, and M. Lakshmanan, "Observation and characterization of chimera states in coupled dynamical systems with nonlocal coupling," Physical Review E, vol. 89, Article ID 52914, 2014.

[49] M. Mikhaylenko, L. Ramlow, S. Jalan, and A. Zakharova, "Weak multiplexing in neural networks: switching between chimera and solitary states," Chaos: An Interdisciplinary Journal of Nonlinear Science, vol. 29, no. 2, Article ID 23122, 2019.

[50] S. Majhi, T. Kapitaniak, and D. Ghosh, "Solitary states in multiplex networks owing to competing interactions," Chaos: An Interdisciplinary Journal of Nonlinear Science, vol. 29, no. 1, Article ID 13108, 2019. 